\title{
A Survey for Low Surface Brightness Dwarf Galaxies Around M31
}

\author{
Taft E. Armandroff \\ Kitt Peak National Observatory, National Optical Astronomy \\ Observatories, ${ }^{1}$ P.O. Box 26732, Tucson, AZ 85726
}

James E. Davies ${ }^{2}$

Department of Astronomy, University of Wisconsin, Madison, WI 53706

\author{
George H. Jacoby \\ Kitt Peak National Observatory, National Optical Astronomy \\ Observatories, ${ }^{1}$ P.O. Box 26732, Tucson, AZ 85726
}

\begin{abstract}
By applying a digital filtering technique to $1550 \mathrm{deg}^{2}$ of the POSS-II in the vicinity of M31, we found two previously unidentified very low surface brightness dwarf galaxies which we designate And $V$ and VI. Follow-up imaging with the KPNO 4-m telescope resolved these into stars easily. The $V$ - and $I$ - band images of And $V$ indicate a distance similar to that of $\mathrm{M} 31$, and $\langle[\mathrm{Fe} / \mathrm{H}]\rangle \sim-1.5$. All evidence strongly supports its classification as a dwarf spheroidal companion to M31. Data for And VI are being analyzed, but preliminary indications support a similar conclusion. Our search for more dwarfs, including follow-up observations of numerous candidates found via digital filtering, is incomplete; thus, further identifications may be forthcoming.
\end{abstract}

\section{Introduction}

In order to understand many aspects of galaxy formation and galaxy evolution, a complete and unbiased census of the members of the Local Group is necessary. Some examples of the problems that can be addressed with such knowledge include: 1) Accurately defining the faint end of the galaxy luminosity function for the Local Group, allowing comparison with that in rich clusters of galaxies, for example. 2) Identifying additional dynamical probes within the Local Group in order to facilitate deciphering its dynamical history and total mass.

A survey for low surface brightness dwarf galaxies in the direction of M31 yields additional benefits. With a complete census of M31's dwarf companions

\footnotetext{
${ }^{1}$ The National Optical Astronomy Observatories are operated by the Association of Universities for Research in Astronomy, Inc., under cooperative agreement with the National Science Foundation.

${ }^{2}$ Currently at Dept. of Physics and Astronomy, Johns Hopkins University, Baltimore, MD 21218.
} 
and knowledge of their properties, we can compare M31's satellite system with that of the Galaxy. Any differences or similarities between the two systems yield information on the effects of environment on the formation and evolution of dwarf galaxies. In addition, the properties of M31's dwarf companions tell us how much we can generalize from the Galaxy's dwarf satellite system.

The study of M31's dwarf spheroidal companions began with van den Bergh's (1972a, 1974) search of $\sim 700$ square degrees around M31 using IllaJ photographic plates taken with the Palomar 48 -inch Schmidt telescope. By visually examining the plates, he identified three dwarf spheroidal candidates (And I, II $\&$ III). By taking deeper plates with the Palomar 5-m telescope, van den Bergh (1972b, 1974) found that And I, II \& III resolve into stars at approximately the same magnitude as M31's companion NGC 185, strengthening the association of these galaxies with M31. Recent color-magnitude diagrams for And I, II \& III from large terrestrial telescopes (Mould \& Kristian 1990; Armandroff et al. 1993; Konig et al. 1993) and Hubble Space Telescope (Da Costa et al. 1996) have yielded distances that place And I, II \& III in the outer halo of M31. These deep color-magnitude diagrams have also resulted in mean metallicity, metallicity dispersion, and age information. Surface photometry and structural parameters are available for And I, II \& III from Caldwell et al. (1992). All available data suggest that And I, II \& III closely resemble the Galactic dwarf spheroidals.

A number of arguments suggest that a new search of M31's environs for low surface brightness dwarfs may be worthwhile. First, the absolute magnitudes of And I, II \& III do not extend as faint as the Galactic dwarf spheroidals (see Figure 2 of Armandroff 1994), suggesting that perhaps fainter dwarfs exist below the detection limit. Second, the area surveyed by van den Bergh $(1972 a, 1974)$ does not reach the projected distance from the center of M31 that one would expect based on the most distant Galactic dwarf companions (see Figure 6 of Armandroff 1994). Finally, why does M31 have three known dwarf spheroidal companions when the Galaxy has nine? Is this the result of incompleteness in the case of M31 or some intrinsic difference between the two systems?

Consequently, we have undertaken a new search for low surface brightness dwarf galaxies around M31. Our search uses digital filtering techniques applied to the Second Palomar Sky Survey (POSS-II). Our search is complementary to the nearby dwarf galaxy survey by Irwin (1994) that used star counts over much of the sky and revealed the Sextans dwarf spheroidal. Because dwarf galaxies at the M31 distance do not resolve into stars on survey plates, our search is not based on star counts. Since M31 is a northern object, our search is also complementary to that of Whiting et al. (1997) based on visual examination of the southern sky survey plates that called attention to the Antlia dwarf.

\section{Search Strategy}

The POSS-II (Reid et al. 1991) has higher resolution and extends substantially deeper than its first generation counterpart. The availability of the POSS-II in digital form (Lasker \& Postman 1993) enables the use of digital processing techniques and allows full areal coverage around M31. Our survey program employs the POSS-II and seeks to: 1) search an area around M31 that is commensurate with the radial distribution of dwarf spheroidals around the Galaxy; 2) use digi- 
tal processing techniques to enhance the detection of the faintest, lowest surface brightness dwarf spheroidals.

Our search methodology employs a matched filter and is described fully in Armandroff, Davies \& Jacoby (1998). Briefly, the procedure includes: dividing each plate into overlapping 1 square degree regions; fitting a surface to remove the background; removing stars by clipping values $>0.75 \sigma$ above the median; applying a spatial median filter $(77 \times 77$ arcsec; based on And II \& III); visual examination of the processed and original images; selection of candidates that resemble And II \& III on these images. Obvious bright-star ghosts and "optical cirrus" clouds are avoided in the selection process. We check the coordinates against SIMBAD and NED to eliminate known objects. To date, we have applied our detection procedure to 1550 square degrees of the POSS-II around M31.

As the next step in our survey, all the remaining candidates are imaged with the KPNO $0.9-\mathrm{m}$ telescope. These CCD images eliminate any candidates that are plate-based false detections (e.g., remaining bright-star ghosts, unrecognized emulsion problems) and most astronomical misidentifications (e.g., distant galaxy clusters, background low surface brightness spirals, "optical cirrus" clouds). If a candidate shows incipient resolution into stars on the 0.9-m CCD images, then it is probably a nearby dwarf galaxy, so imaging with a 4 -m-class telescope is undertaken. At the time that this paper was written (October 1998), we had not yet surveyed all the POSS-II plates in our desired search area, nor had we completed CCD imaging of our list of candidates from the POSS-II.

\section{Andromeda V}

Thus far, two candidates found by our survey have been resolved into stars (see Figure 1 for POSS-II images of these candidates). The first of these, called Andromeda $\mathrm{V}$, is discussed extensively in Armandroff et al. (1998). We summarize our most important And $\mathrm{V}$ results below.

And $\mathrm{V}$ is located at the following coordinates: $\alpha=1^{\mathrm{h}} 10^{\mathrm{m}} 17.1^{\mathrm{s}}, \delta=+47^{\circ}$ $37^{\prime} 41^{\prime \prime}$ (J2000.0). And V was observed with the KPNO 4-m telescope and primefocus CCD imager in the $V$ and $I$ filters, plus in $\mathrm{H} \alpha$ narrow-band and $R$. In the broad-band filters, And $V$ resolves nicely into stars and exhibits a smooth stellar distribution (Figure 2). In these images, And V resembles the other M.31 dwarf spheroidals. And $V$ does not exhibit the features of classical dwarf irregulars, such as obvious regions of star formation or substantial asymmetries in its stellar distribution. In the And $\mathrm{V}$ continuum-subtracted $\mathrm{H} \alpha$ image, no diffuse $\mathrm{H} \alpha$ emission or $\mathrm{H}$ il regions are detected. The lack of $\mathrm{H} \alpha$ emission in And $\mathrm{V}$ reinforces the conclusion, based on And V's appearance on the broad-band images, that it is a dwarf spheroidal galaxy rather than a dwarf irregular.

And $V$ is not detected in any of the IRAS far-infrared bands either. Because far-infrared emission traces warm dust, and because some Local Group dwarf irregular galaxies are detected by IRAS, And V's lack of far-infrared emission serves as additional, weaker evidence that it is a dwarf spheroidal. No information is currently available about the $\mathrm{H}$ I content of And V via $21 \mathrm{~cm}$ observations. Either an $\mathrm{H}$ I detection or a strict upper limit would be valuable.

We have measured And V's apparent central surface brightness via largeaperture photometry in its core: $25.7 \mathrm{mag} / \operatorname{arcsec}^{2}$ in $V$. And $V$ has a fainter 


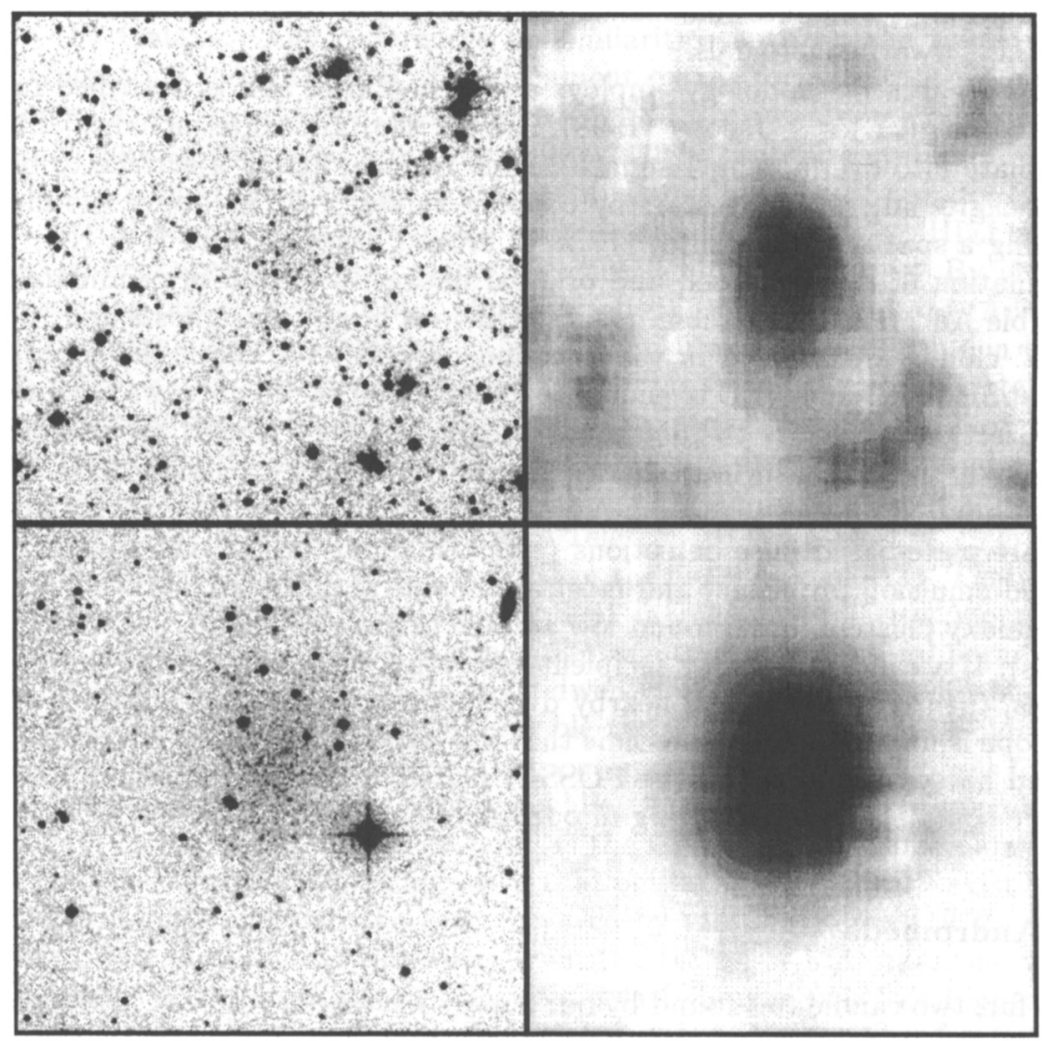

Figure 1. The left panels show images of And V \& VI from the digitized POSS-II; the right panels show the results of applying our digital enhancement procedure. Each panel is 8.5 arcmin on a side.

apparent central surface brightness than And I, II \& III $(24.9,24.8$, and 25.3 $\mathrm{mag} / \operatorname{arcsec}^{2}$ in $V$, respectively; Caldwell et al. 1992). And V probably eluded detection until now due to its very dim apparent surface brightness.

We used our $V$ and $I$ images and photometric standard observations to construct a color-magnitude diagram for And $V$ stars, in order to reveal And V's distance and stellar populations characteristics. Color-magnitude diagrams for the parts of the images dominated by And V stars reveal a red giant branch, which is absent in the outer regions of the images (see Figure 3). The tip of the red giant branch is well defined in the color-magnitude diagram and in the luminosity function. A distance has been derived for And $\mathrm{V}$ based on the $I$ magnitude of the tip of the red giant branch (Da Costa \& Armandroff 1990, Lee et al. 1993). On the distance scale of Lee et al. (1990), the resulting And $\mathrm{V}$ distance is $810 \pm 45 \mathrm{kpc}$. How does this And V distance compare with that of M31? The most directly comparable distance determinations for M31, based on either red giant branch tip stars or RR Lyraes in the M31 halo or horizontal 


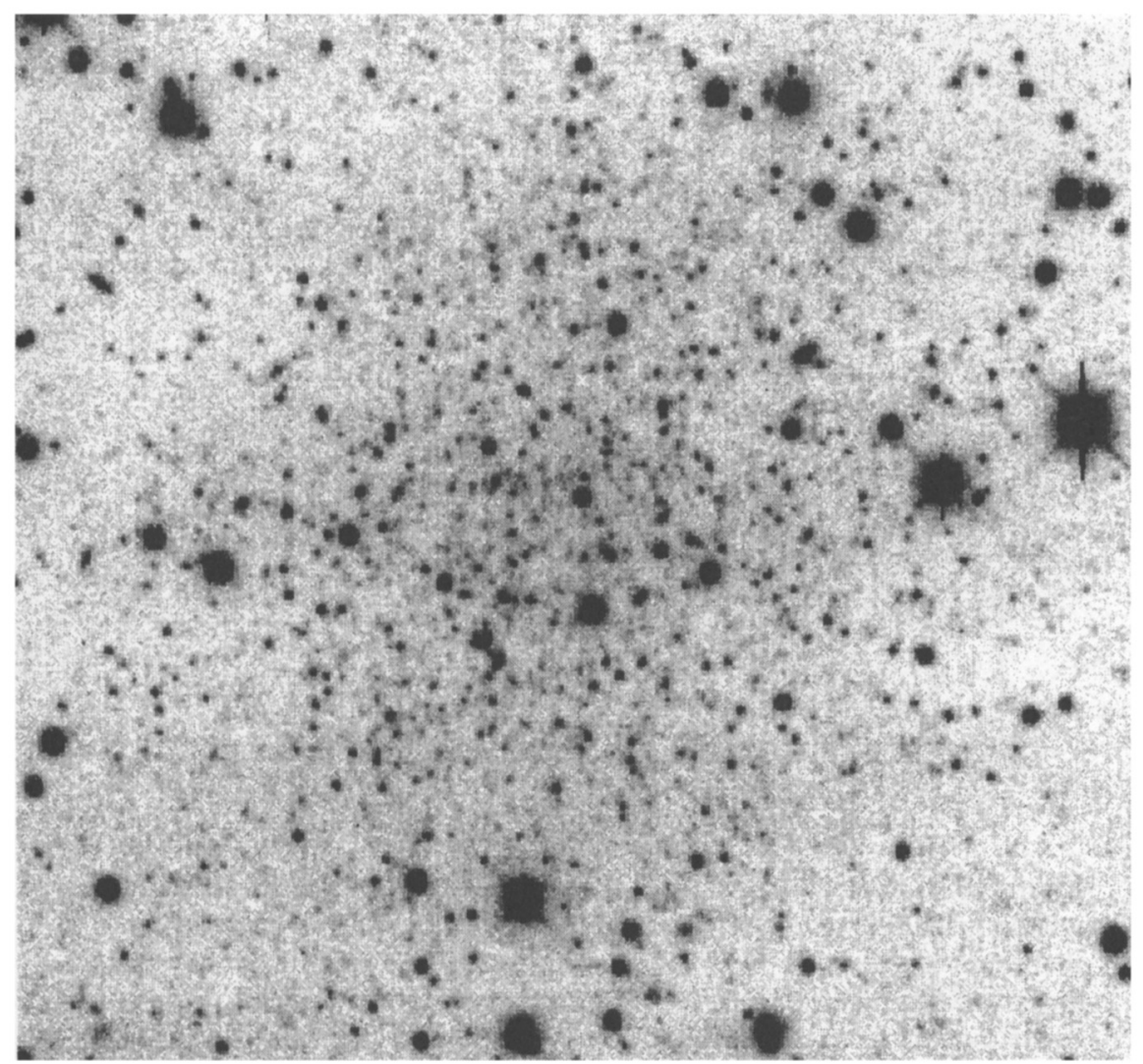

Figure 2. A $V$-band image of And $V$ (three 900-second exposures) taken with the KPNO 4-m telescope. North is at the top, and east is to the right. The image covers $3 \times 3$ arcmin.

branch stars in M31 globular clusters, are $760 \pm 45 \mathrm{kpc}$ or $850 \pm 20 \mathrm{kpc}$, both on the same scale (see Da Costa et al. 1996). Our distance for And V of $810 \pm$ $45 \mathrm{kpc}$ implies that And $\mathrm{V}$ is located at the same distance along the line of sight as M31 to within the uncertainties. And V's projected distance from the center of M31 is $112 \mathrm{kpc}$; And I, II \& III have projected M31-centric distances of 46, 144 and $69 \mathrm{kpc}$, respectively. The above line-of-sight and projected distances strongly suggest that And $\mathrm{V}$ is indeed associated with M31.

In order to investigate the stellar populations in And V, we have compared its color-magnitude diagram with fiducials representing the red giant branches of Galactic globular clusters that span a range of metal abundance (Da Costa \& Armandroff 1990; see Figure 3). Based on the position of the And V giant branch relative to the globular cluster fiducials, the mean metal abundance of And $\mathrm{V}$ is approximately -1.5 . This metallicity is normal for a dwarf spheroidal (e.g., see Figure 9 of Armandroff et al. 1993). No bright blue stars are present 


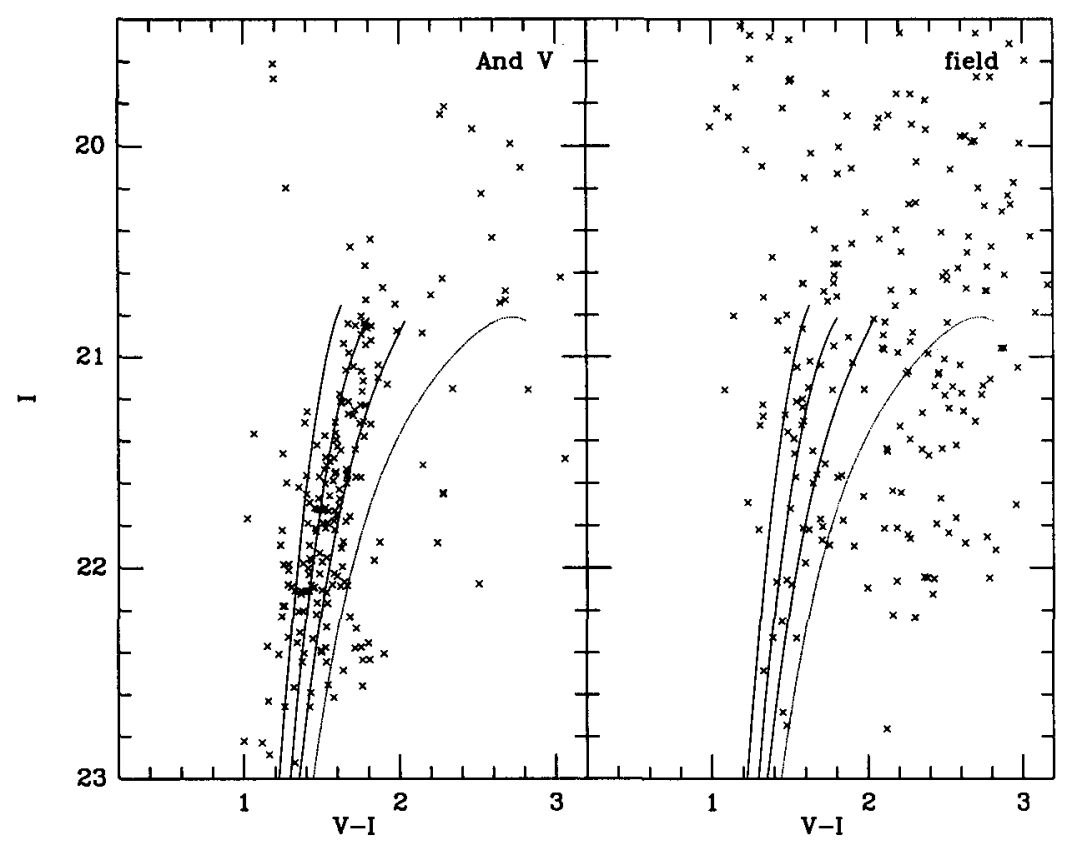

Figure 3. Color-magnitude diagrams. The left panel shows stars within a radius of 71 arcsec of the center of And $V$, where And $V$ members outnumber field contamination. The right panel displays stars greater than 168 arcsec from the center of And V, where the contribution from And $\mathrm{V}$ stars is negligible. The right panel represents 5.5 times more area on the CCD than the left panel. Red giant branch fiducials for four Galactic globular clusters that span a range of metal abundance (Da Costa \& Armandroff 1990), shifted to the distance modulus and reddening of And V, have been overplotted. From left to right, the red giant branch fiducials are M15 $([\mathrm{Fe} / \mathrm{H}]=-2.17)$, M2 (-1.58), NGC $1851(-1.16)$, and 47 Tuc $(-0.71)$.

in the And V color-magnitude diagram. Interpreting via isochrones, this lack of blue stars rules out any stars younger than $200 \mathrm{Myr}$ in And V and is further evidence that And $V$ is a dwarf spheroidal and not a dwarf irregular.

From the luminosities and numbers of upper asymptotic giant branch stars in a metal-poor stellar system, one can infer the age and strength of its intermediate age component (Renzini \& Buzzoni 1986). Using the And V field-subtracted $I$ luminosity function, there is no evidence for upper asymptotic giant branch stars that are more luminous than and redward of the red giant branch tip. Therefore, And V does not have a prominent intermediate age population; in this sense, it is similar to And I \& III. 


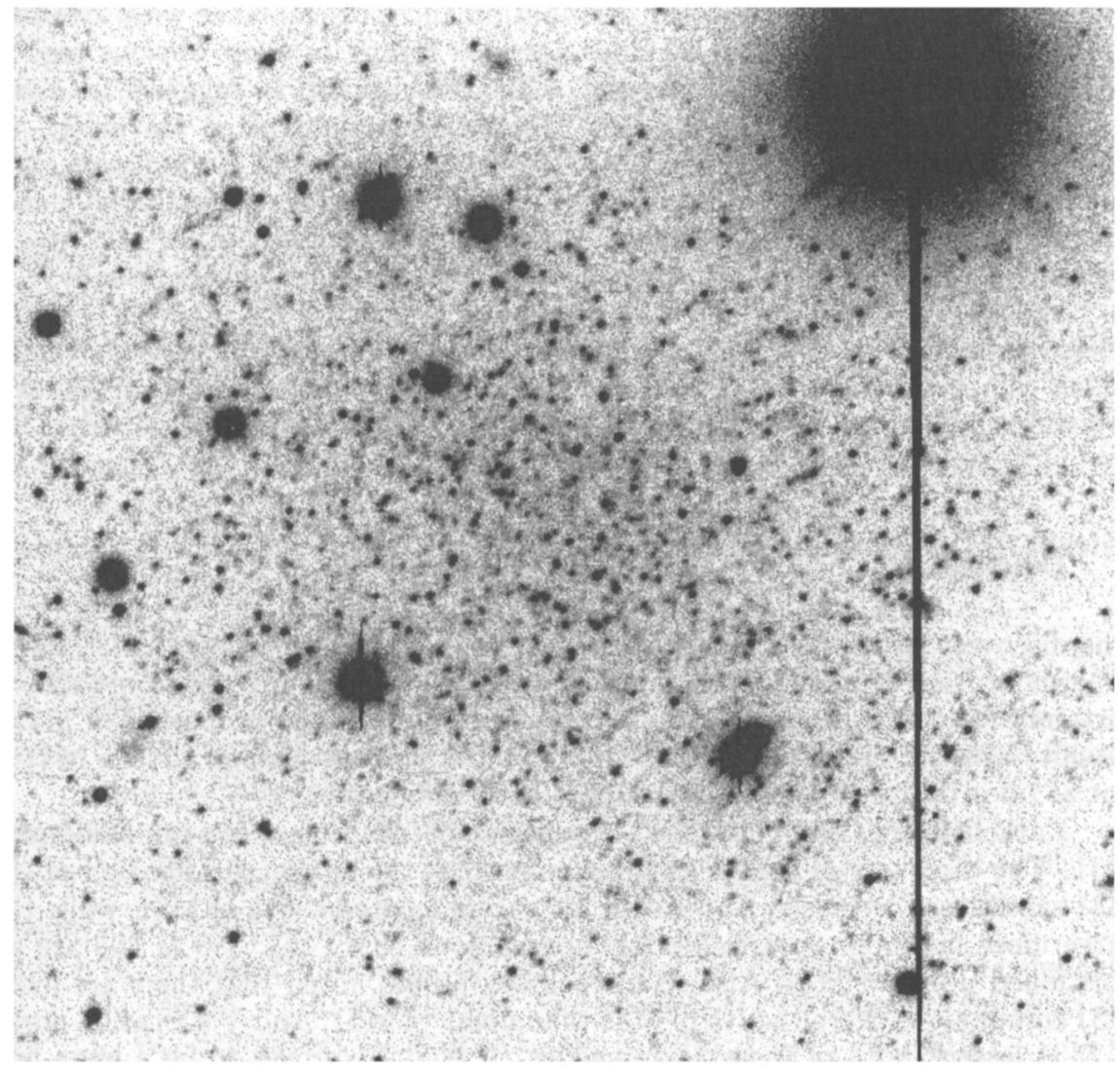

Figure 4. An $R$-band image of And VI (three 300 -second exposures) taken with the KPNO 4-m telescope. North is to the left, and east is at the bottom. The image covers $3.9 \times 3.7$ arcmin.

\section{Andromeda VI}

Using our search methodology, we found a second candidate dwarf spheroidal galaxy, designated Andromeda VI, at the following celestial coordinates: $\alpha=$ $23^{\mathrm{h}} 51^{\mathrm{m}} 46.9^{\mathrm{s}}, \delta=+24^{\circ} 34^{\prime} 45^{\prime \prime}$ (J2000.0). And VI was imaged with the KPNO 4-m telescope prime-focus CCD on 1998 January 23 in $V$ for 300 seconds. And VI resolved nicely into stars in this short $V$ image, suggesting that it is indeed a nearby dwarf galaxy. Several months after we had confirmed to ourselves that And VI is a bona fide nearby dwarf galaxy, we learned that Karachentsev \& Karachentseva were reporting the discovery of this same galaxy, calling it the Pegasus Dwarf (see Brinks \& Grebel 1998).

On 1998 July 15, And VI was imaged more deeply with the KPNO 4-m telescope through the $R$ and $\mathrm{H} \alpha$ filters. The $R$ image of And VI, displayed in 
Figure 4, exhibits a smooth stellar distribution and a resemblance with the other M31 dwarf spheroidals. And VI does not look lumpy or show obvious regions of star formation that would suggest a dwarf irregular, as opposed to dwarf spheroidal, classification. In the And VI continuum-subtracted $\mathrm{H} \alpha$ image, no diffuse $\mathrm{H} \alpha$ emission or $\mathrm{H}$ II regions are detected. The lack of $\mathrm{H} \alpha$ emission rules out current high-mass star formation in And VI and serves as further evidence that And VI is a dwarf spheroidal. Like And V and the other M31 dwarf spheroidals, And VI is not detected in any of the IRAS far-infrared bands.

We are currently working with images of And VI from the WIYN $3.5-\mathrm{m}$ telescope in $B, V$, and $I$. A deep color-magnitude diagram is being constructed which will reveal the distance and stellar populations characteristics of And VI. We will report all our findings on And VI in an upcoming journal paper.

\section{Discussion}

The discovery of the M31 dwarf spheroidal And V and the probable M31 dwarf spheroidal And VI increases the number of M31 dwarf spheroidals from three to certainly four, and probably five. It changes somewhat the properties of M31's satellite system, as discussed below. The most obvious change is that M31 is not as poor in dwarf spheroidals as previously thought.

Karachentsev (1996) discussed the spatial distribution of the companions to M31. The discovery of And V changes somewhat the spatial distribution of the M31 satellites. Curiously, And I, II \& III are all located south of M31, while the three more luminous dwarf elliptical companions NGC 147, 185 \& 205 are all positioned north of M31. Also, Karachentsev (1996) noted that there are more M31 companions overall south of M31 than north of M31. And V's location north of M31 lessens both of these asymmetries. If And VI proves to be a bona fide M31 companion, its location south of M31 would contribute to the asymmetry. With a projected radius from the center of M31 of $112 \mathrm{kpc}$, And V increases slightly the mean projected radius of the M31 dwarf spheroidals from $86 \mathrm{kpc}$ to $93 \mathrm{kpc}$. If And VI is also an M31 dwarf spheroidal, with its projected distance from M31 of $271 \mathrm{kpc}$, the mean projected radius of the M31 dwarf spheroidals increases to $128 \mathrm{kpc}$.

The discovery of nearby dwarf galaxies like And $\mathrm{V}$ augments the faint end of the luminosity function of the Local Group. We do not yet have a reliable $M_{V}$ value for And V, but it appears to be similar to that of And III $\left(M_{V}=\right.$ $-10.2)$ since they have similar extinction-corrected central surface brightness. From a survey of nine clusters of galaxies, Trentham (1998) derived a composite luminosity function that is steeper at the faint end than that of the Local Group (see his Figure 2). He attributed the difference to poor counting statistics and/or incompleteness among the Local Group sample. The discovery of And V reduces slightly the discrepancy between the Local Group luminosity function and the extrapolation of Trentham's (1998) function. If And VI is a bona fide M31 dwarf spheroidal, it also reduces this discrepancy.

It is also of interest to learn whether dwarf spheroidals as faint as Draco and Ursa Minor $\left(M_{V} \approx-9\right)$ exist around M31. By completing our survey and by understanding our completeness limits, we plan to address this question. 


\section{References}

Armandroff, T. E. 1994, in ESO/OHP Workshop on Dwarf Galaxies, ed. G. Meylan \& P. Prugniel (Garching: ESO), 211

Armandroff, T. E., Da Costa, G. S., Caldwell, N., \& Seitzer, P. 1993, AJ, 106, 986

Armandroff, T. E., Davies, J. E., \& Jacoby, G. H. 1998, AJ, 116, 2287

Brinks, E., \& Grebel, E. 1998, Dwarf Tales Newsletter, Number 3

Caldwell, N., Armandroff, T. E., Seitzer, P., \& Da Costa, G. S. 1992, AJ, 103 , 840

Da Costa, G. S., \& Armandroff, T. E. 1990, AJ, 100, 162

Da Costa, G. S., Armandroff, T. E., Caldwell, N., \& Seitzer, P. 1996, AJ, 112, 2576

Irwin, M. J. 1994, in ESO/OHP Workshop on Dwarf Galaxies, ed. G. Meylan \& P. Prugniel (Garching: ESO), 27

Karachentsev, I. 1996, A\&A, 305, 33

Konig, C. H. B., Nemec, J. M., Mould, J. R., \& Fahlman, G. G. 1993, AJ, 106, 1819

Lasker, B. M., \& Postman, M. 1993, in ASP Conf. Ser. 43, Sky Surveys: Protostars to Protogalaxies, ed. B. T. Soifer (San Francisco: ASP), 131

Lee, M. G., Freedman, W. L., \& Madore, B. F. 1993, ApJ, 417, 553

Lee, Y.-W., Demarque, P., \& Zinn, R. 1990, ApJ, 350, 155

Mould, J., \& Kristian, J. 1990, ApJ, 354, 438

Reid, I. N., Brewer, C., Brucato, R. J., McKinley, W. R., Maury, A., Mendenhall, D., Mould, J. R., Mueller, J., Neugebauer, G., Phinney, J., Sargent, W. L. W., Schombert, J., \& Thicksten, R. 1991, PASP, 103, 661

Renzini, A., \& Buzzoni, A. 1986, in Spectral Evolution of Galaxies, ed. C. Chiosi \& A. Renzini (Dordrecht: Reidel), 195

Trentham, N. 1998, MNRAS, 294, 193

van den Bergh, S. 1972a, ApJ, 171, L31

van den Bergh, S. 1972b, ApJ, 178, L99

van den Bergh, S. 1974, ApJ, 191, 271

Whiting, A. B., Irwin, M. J., \& Hau, G. T. 1997, AJ, 114, 996 